\title{
Breached pairing superfluidity: Possible realization in QCD
}

\author{
Elena Gubankova, W. Vincent Liu and Frank Wilczek \\ Center for Theoretical Physics, Department of Physics, \\ Massachusetts Institute of Technology, Cambridge, MA 02139
}

\begin{abstract}
We propose a wide universality class of gapless superfluids, and analyze a limit that might be realized in quark matter at intermediate densities. In the breached pairing color superconducting phase heavy $s$-quarks, with a small Fermi surface, pair with light $u$ or $d$ quarks. The groundstate has a superfluid and a normal Fermi component simultaneously. We expect a second order phase transition, as a function of increasing density, from the breached pairing phase to the conventional color-flavor locked (CFL) phase.
\end{abstract}

Because the primary one-gluon exchange interaction between high-momentum quarks is attractive for quarks in the color antisymmetric $\overline{\mathbf{3}}$ channel, it is a firm prediction of QCD that cold dense quark matter is a color superconductor. At asymptotic densities (ignoring the $c, b$ and $t$ quarks) the ground state is well understood: quarks of all three flavors $u, d$, and $s$, pair according to the BCS mechanism, forming the color-flavor locked (CFL) phase [1].

It is much less clear what QCD predicts for the ground state at subasymptotic densities, which could be relevant for describing neutron stars. Differences among the quark masses cause mismatches among the Fermi surfaces of the species which potentially pair. There is no longer an abundance of degenerate low-energy particle-particle or hole-hole states with opposite momenta both near their Fermi surfaces, and so it is less obvious what modes are the best candidates for coherent alignment by attractive interactions.

One much-discussed possibility is the LOFF phase (Larkin-Ovchinnikov-Fulde-Ferrel 2]). In the context of QCD these ideas lead to the crystalline color superconductivity [3]. Here we suggest quite a different possibility for ordering with mismatched Fermi surfaces, that might be realized at intermediate densities in QCD.

In QCD at intermediate densities, $\mu \sim 200-300 \mathrm{MeV}$, there is not only mismatch in quark Fermi surfaces but also a different dispersion relation, since the heavy $s$ quark, unlike the light $u$ and $d$ quarks, needs not be ultra-relativistic. This difference makes plausible a pairing phase, wherein strange quarks are raised to higher kinetic energies to exploit favorable possibilities for correlation energy through pairing. The opposite limit pairing of a heavy and light species when heavy species has the larger Fermi surface - is the arena for the interior gap phase discussed in [4].

For illustrative purposes we analyze a toy model with a massive $s$ and a massless $u$ quark. The Fermi momenta are related to chemical potentials as

$$
p_{F}^{u}=\mu-\delta \mu^{e}, \quad p_{F}^{s}=\sqrt{\left(\mu+\delta \mu^{e}\right)^{2}-m_{s}^{2}}
$$

where $\delta \mu^{e}$ will be tuned to enforce number equality (a stand-in for electric neutrality). We are interested in the case when the Fermi momentum for the $s$-quark is smaller than that for the $u$-quark, $p_{F}^{s}<p_{F}^{u}$. For simplicity, we shall linearize the $s$ quark dispersion near its Fermi surface. We have checked that this simplification does not alter our results qualitatively. Our simplified model then has dispersion relations

$$
\varepsilon_{\mathbf{p}}^{u}=V^{u}\left(p-p_{F}^{u}\right), \quad \varepsilon_{\mathbf{p}}^{s}=V^{s}\left(p-p_{F}^{s}\right)
$$

with $V^{s}<V^{u}$.

In promoting particles of the heavy species to pair around the large Fermi surface of light species, there are two competing energetic factors to consider. These are the single-paticle energy cost of such promotion, $V^{s}\left|p_{F}^{u}-p_{F}^{s}\right|$ per pair, versus the gain from creating a pair, $\kappa\left(V^{u}+V^{s}\right)$, where $\kappa$ is the momentum gap. There is a net profit when

$$
\left|p_{F}^{u}-p_{F}^{s}\right|<\kappa \frac{V^{u}+V^{s}}{V^{s}} .
$$

For flat dispersion for the $s$-quark, $V^{s} \ll V^{u}$, promotion of $s$-quarks to a higher $u$-quark Fermi surface does not cost much energy, and a paired phase is favored. Translating this into possible effects of non-zero $s$-quark mass the allowed range for pairing is given by

$$
\left|\delta \mu^{e}-\frac{m_{s}^{2}}{4 \mu}\right|<\Delta \frac{V^{u}}{V^{s}} .
$$

It can be more economical to promote heavy particles to higher Fermi momentum than to equalize the two Fermi surfaces by deforming both. In earlier work [4] we suggested the terminology "interior gap" for this phenomenon, motivated by the peaking of the gap parameter interior to the large Fermi surface that carries most of the spectral weight for gapless modes. Following that thought, it would be natural to call the limit we are analyzing here "exterior gap". But to emphasize the common physical mechanism, we use "breached pairing" to cover both. The pairing is breached, in that it vanishes in a solid annulus in momentum space.

To allow quantitative estimates in analytic form we consider a schematic model involving pairing of $u$ and $s$ quarks. Starting with non-interacting degenerate Fermi 
gases of $u$ and $s$-quarks, turn on a weak attractive interaction between light and heavy species with a coupling $-g<0$. In a basis of light particles and heavy holes the quadratic part of the Hamiltonian is

$$
\mathcal{H}_{\text {quad }}=\left(\begin{array}{ll}
\psi_{u \mathbf{p}}^{\dagger} & \psi_{s-\mathbf{p}}
\end{array}\right)\left(\begin{array}{cc}
\varepsilon_{\mathbf{p}}^{u} & -\Delta^{*} \\
-\Delta & -\varepsilon_{\mathbf{p}}^{s}
\end{array}\right)\left(\begin{array}{c}
\psi_{u \mathbf{p}} \\
\psi_{s-\mathbf{p}}^{\dagger}
\end{array}\right),
$$

where the gap parameter is defined as $\Delta=$ $g(2 \pi)^{-3} \int d^{3} \mathbf{p}\left\langle\psi_{u \mathbf{p}}^{\dagger} \psi_{s-\mathbf{p}}^{\dagger}\right\rangle_{B P}$, where the groundstate is assumed to be the breached pairing state. $\mathcal{H}_{\text {quad }}$ can be diagonalized by the Bogoliubov transformation

$$
\left(\begin{array}{c}
\psi_{u \mathbf{p}} \\
\psi_{s-\mathbf{p}}^{\dagger}
\end{array}\right)=\left(\begin{array}{cc}
\cos \theta_{\mathbf{p}} & -\sin \theta_{\mathbf{p}} \\
\sin \theta_{\mathbf{p}} & \cos \theta_{\mathbf{p}}
\end{array}\right)\left(\begin{array}{c}
\tilde{\psi}_{u \mathbf{p}} \\
\tilde{\psi}_{s-\mathbf{p}}^{\dagger}
\end{array}\right)
$$

with $\sin 2 \theta_{\mathbf{p}}=\Delta / \sqrt{\varepsilon_{\mathbf{p}}^{+2}+\Delta^{2}}$. The new fermion fields $\tilde{\psi}$ define the form of the interior gap wavefunction up to a relative phase (due to degeneracy) by $\tilde{\psi}|0\rangle_{B P}=0$. In the meantime, we obtain two branches of quasi-particle excitations with the spectra $E_{\mathbf{p}}^{ \pm}=\varepsilon_{\mathbf{p}}^{-} \pm \sqrt{\varepsilon_{\mathbf{p}}^{+2}+\Delta^{2}}$, where $\varepsilon_{\mathbf{p}}^{ \pm}=\frac{1}{2}\left(\varepsilon_{\mathbf{p}}^{u} \pm \varepsilon_{\mathbf{p}}^{s}\right)$. The order parameter is related to $\theta_{\mathbf{p}}$ by $\left\langle\psi_{u \mathbf{p}}^{\dagger} \psi_{s-\mathbf{p}}^{\dagger}\right\rangle_{B P}=\frac{1}{2} \sin 2 \theta_{\mathbf{p}}$ outside the breached region.

The energy of the breached pairing state $|0\rangle_{B P}$ can be computed from the diagonalized Hamiltonian as

$$
\langle H\rangle_{B P}=\frac{1}{g} \Delta^{2}-\int_{D} \frac{d^{3} \mathbf{p}}{(2 \pi)^{3}}\left(\varepsilon_{\mathbf{p}}^{-}+\sqrt{\varepsilon_{\mathbf{p}}^{+2}+\Delta^{2}}\right) .
$$

The first term is the mean field potential and the integration is restricted to the area $D$ defined by $\left(p_{0}-\lambda\right) \leq$ $|\mathbf{p}| \leq p_{\Delta}^{-}$and $p_{\Delta}^{+} \leq|\mathbf{p}| \leq\left(p_{0}+\lambda\right)$, where $p_{\Delta}^{ \pm}$are the two roots of $E_{\mathbf{p}}^{+}=0, p_{0}$ is the momentum defined by $\varepsilon_{\mathbf{p}_{0}}^{+}=0$ :

$$
p_{0}=p_{F}^{u}-\delta p_{F} \frac{V^{s}}{V^{u}+V^{s}},
$$

and $\lambda$ is the ultraviolet cutoff. (We will always be assuming $p_{0} \gg \lambda \gg \Delta, \delta p_{F}$.) Thus

$$
p_{\Delta}^{ \pm}=p_{F}^{u}-\frac{1}{2} \delta p_{F} \pm \frac{1}{2} \sqrt{\delta p_{F}^{2}-\frac{4 \Delta^{2}}{V^{u} V^{s}}}
$$

with $\delta p_{F} \equiv p_{F}^{u}-p_{F}^{s}>0$. Varying the groundstate energy with respect to $\Delta, d\langle H\rangle_{B P} / d \Delta=0$, we find the integral equation for the gap parameter

$$
1=g \int_{D} \frac{d^{3} \mathbf{p}}{(2 \pi)^{3}} \frac{1}{2} \frac{1}{\sqrt{\varepsilon_{\mathbf{p}}^{+2}+\Delta^{2}}} .
$$

Note that the integrand peaks near to $|\mathbf{p}|=p_{0}$ where the energy denominator vanishes for $\Delta \rightarrow 0$.

We can illuminate the physical meaning of $p_{\Delta}^{ \pm}$. Pairing of $u$ and $s$ quarks produces two quasiparticle excitation branches. While $E_{\mathbf{p}}^{-}<0$ always holds, the $E_{\mathbf{p}}^{+}$branch changes sign in the region $\left[p_{\Delta}^{-}, p_{\Delta}^{+}\right]$. The negativity of
$E_{\mathbf{p}}^{+}$means that the corresponding states are singly occupied by, in our chosen particle-hole basis, the $u$-quarks. Since this region has single occupation, it does not contribute to the pairing interaction, and it must therefore be excluded from the gap equation integral. The same phenomenon appears in a more formal treatment with Green functions [5]. Integration over energy yields a nonzero contribution only if the two poles of the Bogoliubov quasiparticles reside in different half planes of the whole complex energy plane.

Two cases can arise, depending on whether the $E_{\mathbf{p}}^{+}$ branch can intersects the zero energy axis:

$B C S$ superfluidity. For $\delta p_{F} \leq \frac{2 \Delta}{\sqrt{V^{u} V^{s}}}, E_{\mathbf{p}}^{+}$never intersects $E_{\mathbf{p}}^{+}=0$. Then $E_{\mathbf{p}}^{+}>0$ and $E_{\mathbf{p}}^{-}<0$ for all $\mathbf{p}$. Pairing is possible in the whole range $\left(p_{0}-\lambda\right) \leq|\mathbf{p}| \leq$ $\left(p_{0}+\lambda\right)$. We arrive at the BCS solution for the gap

$$
\Delta=\lambda\left(V^{u}+V^{s}\right) \exp \left(-\frac{1}{N_{+}(0) g}\right),
$$

where $N_{+}(0)=\frac{1}{(2 \pi)^{3}} \int d^{3} \mathbf{p} \delta\left(\epsilon_{\mathbf{p}}^{+}\right)$is the density of states, and $\lambda$ is the ultraviolet cutoff. Since $N_{+}(0) \sim p_{0}^{2}$, the gap parameter decreases as a function of increasing $\delta p_{F}$ with $p_{F}^{u}$ fixed.

Breached pairing (BP) superfluidity. This occurs when $\delta p_{F}>\frac{2 \Delta}{\sqrt{V^{u} V^{s}}}$. $E_{\mathbf{p}}^{+}=0$ has two roots. We integrate below $p_{\Delta}^{-}$and above $p_{\Delta}^{+}$, but exclude the region $p_{\Delta}^{-} \leq|\mathbf{p}|<p_{\Delta}^{+}$. A solution of the gap equation exists only if $g>g_{c}$, with the critical coupling constant

$$
g_{c}=\left[N_{+}(0) \ln \left(\frac{\lambda}{\delta p_{F}} \frac{\left(V^{u}+V^{s}\right)}{\sqrt{V^{u} V^{s}}}\right)\right]^{-1} .
$$

For $V^{s} / V^{u} \ll 1$ with fixed Fermi momenta, $g_{c} \rightarrow 0$ becomes arbitrarily weak.

Expanding the gap equation (10) for small $\Delta<\delta p_{F}$, we find the critical behavior

$$
\Delta \sim\left(g-g_{c}\right)^{\frac{1}{2}}
$$

with $g_{c}$ defined in Eq. (12).

The quasiparticle excitations of the breached pairing phase differ qualitatively from those of BCS. In the BCS phase, $E_{\mathbf{p}}^{ \pm}$branches are both gapped with their minimum energies given by $\left|\epsilon_{p_{0}}^{-} \pm 2 \Delta \sqrt{V_{u} V_{s}} /\left(V_{u}+V_{s}\right)\right| \neq 0$, respectively. By contrast, in the breached pairing phase $E_{\mathbf{p}}^{+}$intersects zero energy. Only the $E_{p}^{-}$branch is fully gapped.

For finite $\Delta$, the gap equation (10) needs to be solved self-consistently, implementing the restriction in integration region, $|\mathbf{p}| \leq p_{\Delta}^{-}$and $|\mathbf{p}| \geq p_{\Delta}^{+}$, which depends on $\Delta$. To this end, we transform the integral gap equation into an algebraic one by introducing a (BCS) gap paramter, $\Delta_{0} \equiv \Delta\left(\delta p_{F}=0\right)$. The BCS gap $\Delta_{0}$ implicitly defines the interaction strength, $1 / g=$ 
$N_{+}(0) \ln \left[\lambda\left(V^{u}+V^{s}\right) / \Delta_{0}\right]$. We shall use $\Delta_{0}$ to parameterize the interaction strength below. Performing the integrals

$$
\begin{aligned}
& \int_{p_{0}-\lambda}^{p_{0}+\lambda} \frac{d p}{\sqrt{\varepsilon_{\mathbf{p}}^{+2}+\Delta_{0}^{2}}} \\
= & \int_{p_{0}-\lambda}^{p_{0}+\lambda} \frac{d p}{\sqrt{\varepsilon_{\mathbf{p}}^{+2}+\Delta^{2}}}-\int_{p_{\Delta}^{-}}^{p_{\Delta}^{+}} \frac{d p}{\sqrt{\varepsilon_{\mathbf{p}}^{+2}+\Delta^{2}}}
\end{aligned}
$$

where $\varepsilon_{\mathbf{p}}^{+}=\frac{1}{2}\left(V^{u}+V^{s}\right)\left(p-p_{0}\right)$, we obtain

$$
\frac{\Delta_{0}^{2}}{\Delta^{2}}=\frac{\varepsilon_{p_{\Delta}^{+}}^{s}}{\varepsilon_{p_{\Delta}^{-}}^{s}}
$$

for $\lambda \rightarrow \infty$. After some elementary algebra, the above equation reduces to

$$
\frac{\Delta_{0}^{2}}{\Delta^{2}}=\frac{\delta p_{F}+\sqrt{\delta p_{F}^{2}-\frac{4 \Delta^{2}}{V^{u} V^{s}}}}{\delta p_{F}-\sqrt{\delta p_{F}^{2}-\frac{4 \Delta^{2}}{V^{u} V^{s}}}} .
$$

It has a (stable) solution only if

$$
\delta p_{F}<\frac{\Delta_{0}}{\sqrt{V^{u} V^{s}}},
$$

which is equivalent to $g>g_{c}$ (see Eq. (12)). In solving Eq. (16), we distinguish three cases:

I: Chemical potentials fixed. Then $\delta p_{F}$ remains unchanged before and after pairing, and the gap equation permits two solutions:

$$
\Delta= \begin{cases}\Delta_{0}, & (\mathrm{BCS}) \\ {\left[\Delta_{0} \sqrt{V^{u} V^{s}}\left(\delta p_{F}-\delta p_{\text {th }}\right)\right]^{\frac{1}{2}},} & \text { (unstable BP })\end{cases}
$$

where $\delta p_{\text {th }}=\frac{\Delta_{0}}{\sqrt{V^{u} V^{s}}}$ (threshold value). In this case, the BP state always has higher energy than the normal state, so it is unstable (see Eq. (24)). Fig. 10 makes it further clear that even if it is a metastable solution at $\delta p_{F}>\delta p_{\text {th }}$, the BP state always has a smaller $\Delta$ than BCS. It is therefore energetically disfavored. This case was considered by Sarma and others [ [] a long time ago.

II: Overall quark density fixed. In this case one has to adjust the overall chemical potential, $\mu_{o a} \equiv \frac{1}{2}\left(V^{u} p_{F}^{u}+\right.$ $\left.V^{s} p_{F}^{s}\right)=\frac{1}{2}\left(V^{u}+V^{s}\right) p_{0}$, in order to accommodate a fixed overall density when pairing occurs. We hold the relative chemical potential, $\delta \mu=V^{u} p_{F}^{u}-V^{s} p_{F}^{s}$ fixed. The mismatched Fermi momentum is then altered, $\delta p_{F} \rightarrow \delta \tilde{p}_{F}$, with ' $\sim$ ' denoting the new Fermi momenta $\tilde{p}_{F}^{u, s}$ after pairing.

We find $\delta \tilde{p}_{F}$ is determined by

$$
p_{0}^{3}-p_{0}^{2} \frac{\varepsilon_{p_{F}^{u}}^{-}-\varepsilon_{p_{F}^{s}}^{-}}{V^{u}+V^{s}}=\tilde{p}_{0}^{3}-\tilde{p}_{0}^{2} \frac{\tilde{\varepsilon}_{\tilde{p}_{\Delta}^{+}}^{-}-\tilde{\varepsilon}_{\tilde{p}_{\Delta}^{-}}^{-}}{V^{u}+V^{s}},
$$
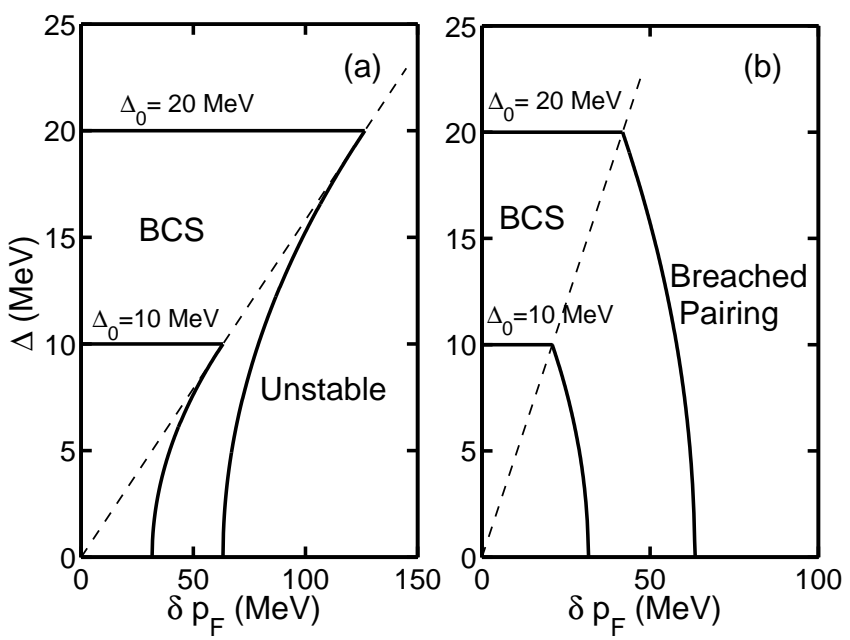

FIG. 1: Solution of the gap equation as a function of the Fermi momentum mismatch $\delta p_{F}$ for different coupling constants parameterized by $\Delta_{0}$. (a) Chemical potentials fixed: The dashed line, $\delta p_{F}=\frac{2 \Delta}{\sqrt{V^{u} V^{s}}}$, marks the transition between the BCS and breached pairing phases. The range for the unstable breached phase is $\frac{\Delta_{0}}{\sqrt{V^{u} V^{s}}} \leq \delta p_{F} \leq \frac{2 \Delta_{0}}{\sqrt{V^{u} V^{s}}}$. (b) The total density fixed: The dashed line, ploted by $\delta p_{F}=\frac{2 \Delta}{\sqrt{V^{u} V^{s}}}\left(1-\alpha^{2}\right)$, marks a continuous transition between the BCS and breached pairing phases. The range for breached pairing is defined by $\frac{2 \Delta_{0}\left(1-\alpha^{2}\right)}{\sqrt{V^{u} V^{s}}} \leq \delta p_{F} \leq \frac{\Delta_{0}}{\sqrt{V^{u} V^{s}}}$. Both (a) and (b) are plotted for $V^{u}=1$ and $V^{s}=0.1$.

where $\tilde{p}_{0}=p_{0}+\frac{2 V^{u} V^{s}}{V^{u}-V^{s 2}}\left(\delta p_{F}-\delta \tilde{p}_{F}\right)$. For large Fermi momenta compared to the mismatch in Fermi surfaces, $\delta p_{F} \ll p_{F}^{u}, p_{F}^{s}$, we find

$$
\delta \tilde{p}_{F}=\frac{\delta p_{F}}{1-2 \alpha^{2} \frac{\Delta^{2}}{\Delta_{0}^{2}+\Delta^{2}}},
$$

where $\alpha \equiv\left(V^{u}-V^{s}\right) /\left(V^{u}+V^{s}\right)$. The gap equation (16) (with $\delta p_{F} \rightarrow \delta \tilde{p}_{F}$ ) and Eq. (20) are solved by

$$
\Delta=\left\{\begin{array}{l}
\Delta_{0}, \\
{\left[\Delta_{0} \sqrt{V^{u} V^{s}}\left(\frac{\delta p_{\mathrm{th}}-\delta p_{F}}{2 \alpha^{2}-1}\right)\right]^{\frac{1}{2}} .}
\end{array}\right.
$$

The breached pairing phase is realized for $2 \alpha^{2}-1>$ 0, i.e., $V^{s} / V^{u}<3-2 \sqrt{2}$. (See Fig. 10.) The dashed line shows a continuous phase transition between BCS $\left(\delta p_{F} \leq \delta p_{F}^{c}\right)$ and breached pairing $\left(\delta p_{F}>\delta p_{F}^{c}\right)$, where $\delta p_{F}^{c}=\frac{2 \Delta_{0}}{\sqrt{V^{u} V^{s}}}\left(1-\alpha^{2}\right)$. There is no overlap region where both states are solutions. The breached pairing state is stable and has lower energy than that of the unpaired matter (see (24) below).

For higher values of $V^{s} / V^{u}$ (but smaller than one), the BP solution becomes unstable, being of the same class as (18). Very close to $2 \alpha^{2}-1=0$ one finds from Eq. (21) that $\delta p_{F}^{c}$ approached $\delta p_{\text {th }}$. The value $3-2 \sqrt{2}$ is model dependent, and will be different when a more realistic model and a more accurate approximation scheme are 
used. In the limit $V^{s} / V^{u} \rightarrow 0$ (so $\alpha \rightarrow 1$ ), the gap equation (16) exhibits the breached pairing phase for all $\delta p_{F}>0$; BCS occurs only at the point $\delta p_{F}=0$.

In mean field theory there is a single second-order phase transition between the BCS and BP phases (Fig [b). At fixed mismatch in Fermi momenta $\delta p_{F}$ and as $\Delta_{0}$ increases, one first encounters $\mathrm{BP}$ and then the BCS phase. The transition between the BP and BCS phases occurs at $\delta p_{F}=\delta p_{F}^{c}$. Since (21) is symmetric with respect to interchange of $V^{u}$ and $V^{s}$, the expression for critical points holds equally for both interior and "exterior" gap limits.

III: Relative quark density fixed. In this case, we also hold the overall chemical potential fixed while allowing the overall density to vary. In the limit $p_{0} \gg \lambda \gg \Delta, \delta p_{F}$, we find

$$
\delta \tilde{p}_{F}=\sqrt{\delta p_{F}^{2}+\frac{4 \Delta^{2}}{V^{u} V^{s}}} .
$$

The gap equation (16) (with $\delta p_{F} \rightarrow \delta \tilde{p}_{F}$ ) has no BCS but BP solution for any $\delta p_{F}>0$,

$$
\Delta=\left[\Delta_{0} \sqrt{V^{u} V^{s}}\left(\delta p_{\mathrm{th}}-\delta p_{F}\right)\right]^{\frac{1}{2}} .
$$

The BCS solution only occurs at a single point, $\delta p_{F}=0$.

Condensation energy. To leading order in $\Delta / \delta p_{F}$, the energy difference between the breached pairing and normal $(\Delta=0)$ phases is

$$
E_{B P}-E_{N}=\left\{\begin{array}{l}
+\frac{N_{+}(0) \Delta^{4}}{2 \delta p_{F}^{2}} \frac{1}{V^{u} V^{s}} \\
-\frac{N_{+}(0) \Delta^{4}}{2 \delta p_{F}^{2}}\left(\frac{2 \alpha^{2}-1}{V^{u} V^{s}}\right) \\
-\frac{N_{+}(0) \Delta^{4}}{2 \delta p_{F}^{2}} \frac{1}{V^{u} V^{s}}
\end{array}\right.
$$

The BP state is energetically favorable in case II if $V^{s} / V^{u}<3-2 \sqrt{2}$ (with overall density and $\delta \mu$ fixed), and it is always favorable in case III (with the relative density and overall $\mu$ fixed).

In the breached pairing scenario pairing occurs at zero total mometum and peaks near the light species Fermi surface. It resembles standard BCS in that the pairing occurs at all angles, there is no necessity for translation or rotation symmetry breaking, and there is a branch of gapped excitations. There are, nevertheless, also gapless modes at two new "effective" Fermi surfaces, between which pairing is suppressed. For small $\Delta$ the energy gain of the breached pairing phase with respect to unpaired normal matter is $\sim \Delta^{4}$, parametrically less than $\sim \Delta^{2}$ in the BCS case.

Discussion. In the context of QCD, enforcing charge neutrality will make case III relevant for the $u$ and $d$ quarks. For pairing between strange and light quarks, case II is relevant. Thus, breached pairing allows all flavors to form condensates $\langle u d\rangle_{B P},\langle u s\rangle_{B P},\langle d s\rangle_{B P}$ of breached pairing type. We suspect that there is a density range where it is energetically favorable compared to unpaired matter or the $2 \mathrm{SC}$ phase with only $\langle u d\rangle_{B C S} \neq 0$ condensation. Alford and Rajagopal [7] showed the absence of 2SC phase in compact stars, and suggested that there should be some non-BCS pattern of pairing including possible unpaired matter in the range of densities between hadronic and CFL matter. The breached pairing version of CFL appears to be a serious candidate for these intermediate densities. Since there is a simple second order phase transition from BP to BCS superfluidity, as a function of increasing density, it is natural to expect a single phase transition from breached pairing CFL to standard CFL quark matter. Detailed calculations, including shifts of Fermi surfaces due to electric and color neutrality, will be required to determine the range, if any, over which breached pairing CFL is favored (in preparation). At strong coupling one could even speculate about breached pairing CFL states at zero strangeness, where the strange chemical potential falls between $\pm m_{s}$.

The possibility that one might rearrange the freeparticle Fermi surfaces to take advantage of opportunities for pairing was considered long ago in condensed matter literature by Sarma and others [6]. Superfluidity with quasiparticle dispersion relation crossing zero, thus leading to gapless modes, was encountered in a model resembling ours in Ref. 8]. In neither case, however, was the possibility of a stable breached pairing state recognized. (See also a recent study in cold atoms 9].) As this work was being completed reference Ref. [10] appeared, in which a related problem, imposing a charge neutrality condition directly, was analyzed.

We have phrased our discussion in terms of QCD and quarks, but one can easily imagine closely related universality classes for condensed matter or cold atom systems.

The authors thank Jeff Bowers for useful discussions and valuable suggestions, Wolfram Schroers for help in numerical analyses; and M. Forbes, J. Kundu, Y. Schroeder and I. Sigalov. This work is supported in part by funds provided by the U.S.Department of Energy (D.O.E.) under cooperative research agreement DFFC02-94ER40818.

[1] M. Alford, K. Rajagopal, and F. Wilczek, Nucl. Phys. B537, 443 (1999).

[2] A. I. Larkin and Yu. N. Ovchinnikov, Sov. Phys. JETP 20, 762 (1965); P. Fulde and R. A. Ferrell, Phys. Rev. 135, A550 (1964).

[3] M. Alford, J. Bowers and K. Rajagopal, Phys. Rev. D 63, 074016 (2001); J. Bowers and K. Rajagopal, Phys. Rev. D 66, 065002 (2002); J. Kundu and K. Rajagopal, Phys. Rev. D 65, 094022 (2002).

[4] W. V. Liu and F. Wilczek, Phys. Rev. Lett. 90, 047002 (2003).

[5] T. Schaefer and F. Wilczek, Phys. Rev. D 60, 074014 (1999); J. A. Bowers, J. Kundu, K. Rajagopal and E. 
Shuster, Phys. Rev. D 64, 014024 (2001).

[6] G. Sarma, Phys. Chem. Solid 24, 1029 (1963); S. Takada and T. Izuyama, Prog. Theor. Phys. 41, 635 (1969).

[7] M. Alford and K. Rajagopal, JHEP 0206, 031 (2002).

[8] M. Alford, J. Berges, and K. Rajagopal, Phys. Rev. Lett.
84, 598 (2000).

[9] S.T. Wu and S. Yip, Phys. Rev. A (to appear). arXiv: cond-mat/0303185.

[10] I. Shovkovy and M. Huang, hep-ph/0302142. 